\title{
ARTICLE OPEN \\ Ser/Thr protein kinase PrkC-mediated regulation of GroEL is critical for biofilm formation in Bacillus anthracis
}

\author{
Gunjan Arora ${ }^{1}$, Andaleeb Sajid ${ }^{1}$, Richa Virmani ${ }^{1}$, Anshika Singhal ${ }^{1}$, C. M. Santosh Kumar ${ }^{2}$, Neha Dhasmana ${ }^{1}$, Tanya Khanna ${ }^{1}$, \\ Abhijit Maji ${ }^{1}$, Richa Misra ${ }^{1}$, Virginie Molle ${ }^{3}$, Dörte Becher ${ }^{4}$, Ulf Gerth ${ }^{4}$, Shekhar C. Mande ${ }^{2}$ and Yogendra Singh ${ }^{1,5}$
}

PrkC is a conserved Ser/Thr protein kinase encoded in Bacillus anthracis genome. PrkC is shown to be important for B. anthracis pathogenesis, but little is known about its other functions and phosphorylated substrates. Systemic analyses indicate the compelling role of PrkC in phosphorylating multiple substrates, including the essential chaperone GroEL. Through mass spectrometry, we identified that PrkC phosphorylates GroEL on six threonine residues that are distributed in three canonical regions. Phosphorylation facilitates the oligomerization of GroEL to the physiologically active tetradecameric state and increases its affinity toward the co-chaperone GroES. Deletion of prkC in B. anthracis abrogates its ability to form biofilm. Overexpression of native GroEL recovers the biofilm-forming ability of prkC deletion strain. Similar overexpression of GroEL phosphorylation site mutants (Thr to Ala) does not augment biofilm formation. Further analyses indicate the phosphorylation of GroEL in diverse bacterial species. Thus, our results suggest that PrkC regulates biofilm formation by modulating the GroEL activity in a phosphorylation-dependent manner. The study deciphers the molecular signaling events that are important for biofilm formation in $B$. anthracis.

npj Biofilms and Microbiomes (2017)3:7 ; doi:10.1038/s41522-017-0015-4

\section{INTRODUCTION}

Bacillus anthracis (B. anthracis) is a bacterial pathogen that causes anthrax and has been historically used as a model organism to understand the bacterial response during infection. ${ }^{1}$ The lifecycle of $B$. anthracis comprises of vegetative and sporulation phases and possess the ability to form capsules and biofilms. ${ }^{2,3} B$. anthracis, Bacillus cereus, and Bacillus thuringiensis are the three major species comprising the pathogenic Bacillus cereus group. These bacteria are difficult to eradicate, both in the environment and during infection, mainly due to the efficient development of spores and biofilms. $B$. anthracis cells readily form biofilms under stagnant conditions in environment and protect the vegetative cells, which continue to divide within biofilm communities. ${ }^{4}$ The cells can eventually sporulate and disseminate, thus causing exponential increase in bacterial cell number under favorable conditions. During infection, pathogens tend to sporulate or form biofilms on epithelial cells, enabling the bacteria to escape innate immune responses and become antibiotic-resistant. ${ }^{5-8}$ Thus, entering the sporulation phase or forming biofilm is a survival strategy for bacteria., ${ }^{3-11}$ The mechanism of biofilm formation remains poorly understood in $B$. anthracis, although biofilm-forming cells are suggested to be particularly resistant to high levels of antibiotic treatments. ${ }^{3}$ Further, Bacillus forms communities that have both biofilm and spores, and the biofilm disassembly leads to spore release. It is plausible that for planktonic cells biofilm-associated growth provides fitness advantage.

Bacterial cells sense extracellular signals and respond in a concerted manner during morphogenesis. The role of molecular signaling events occurring inside $B$. anthracis remains unknown. The cascades initiate at cellular surface and culminate in the modulation of defined set of proteins, leading to specific cellular response, such as biofilm formation. ${ }^{12}$ In $B$. anthracis, PrkC is the only known Ser/Thr protein kinase (STPK) with a sensor domain capable of receiving external signals. ${ }^{13-16}$ PrkC senses muropeptides through the extracellular C-terminal PASTA domains, ${ }^{17-20}$ but its downstream signaling events are not yet known. Homologs of PrkC are conserved in many bacteria such as Mycobacterium, Streptomyces, Staphylococcus, Corynebacterium, and other diverse gram-positive bacteria along with its cognate Ser/Thr phosphatase (PrpC), and is known to regulate vital functions. ${ }^{16,19,21-28}$ PrkC plays an important role in virulence of $B$. anthracis and is important for survival in macrophages during infection. ${ }^{15,}{ }^{16}$ PrkC is capable of transducing signals efficiently during environmental stress conditions and has been proposed to function even in the spores. ${ }^{14,} 17$

In view of these facts, we aimed to explore the importance of $B$. anthracis PrkC in the cellular signaling events. To identify its role(s) in vivo, we searched for proteins phosphorylated by PrkC. Further, using a $B$. anthracis deletion strain of prkC (Bas $\Delta p r k C)$, we investigated its role in regulation of biofilm formation. In accordance to our hypothesis, deletion of prkC resulted in complete loss of biofilm formation and possibly altered cell-tocell adherence properties. Our results indicated that PrkC may regulate biofilm formation by activating an essential chaperone GroEL. Thus, this study describes the novel signaling pathway involving the conserved STPK and chaperone in $B$. anthracis biofilm development.

\footnotetext{
${ }^{1}$ CSIR-Institute of Genomics and Integrative Biology, Delhi 110007, India; ${ }^{2}$ National Centre for Cell Science, NCCS Complex, University of Pune Campus, Ganeshkhind, Pune 411007 Maharashtra, India; ${ }^{3}$ DIMNP, CNRS, University of Montpellier, Montpellier, France; ${ }^{4}$ Institute of Microbiology, Ernst-Moritz-Arndt-University Greifswald, Greifswald D17487, Germany and ${ }^{5}$ Department of Zoology, University of Delhi, Delhi 110007, India

Correspondence: Andaleeb Sajid (sajid.andaleeb@gmail.com) or Yogendra Singh (ysinghdu@gmail.com)

Gunjan Arora and Andaleeb Sajid contributed equally to this work.
}

Received: 23 May 2016 Revised: 29 November 2016 Accepted: 15 December 2016

Published online: 07 March 2017 


\section{RESULTS}

PrkC regulates $B$. anthracis biofilm formation

To understand the effect of PrkC in B. anthracis biofilm formation, we utilized the deletion strain Bas $\Delta p r k C_{1}^{17}$ and compared it with the wild-type Bas-wt. We observed that deletion of prkC did not cause any change in bacterial culture growth rate, as described earlier. $^{15,16}$ However, Bas $\Delta$ prkC cells appeared to have loose settling and surface-adherence property as compared with Bas-wt. Adherence to the growth surface is achieved by forming biofilms at the liquid-air interface. To test the biofilm formation, Bas-wt and $B a s \Delta p r k C$ were cultured under static conditions. We observed that Bas-wt cells were able to form biofilm within 3 days, while this property was lost in Bas $\Delta$ prkC (Fig. 1a, b). To further substantiate this observation, deletion strain was complemented with prkC (Bas $\Delta$ prkC-comp). Biofilm-forming ability of all three strains, Baswt, Bas $\triangle p r k C$, and Bas $\Delta p r k C$-comp, was compared using the quantitative crystal violet assay. Bas $\Delta p r k C$-comp regained the property of biofilm formation to a level similar to Bas-wt (Fig. 1b), indicating that PrkC is involved in biofilm formation. Being a regulatory protein kinase, PrkC exerts its effects through signal transduction pathways involving phosphorylation of its substrates. Thus, changes in biofilm formation may be attributed to PrkCmediated phosphorylation of specific substrate(s), as discussed in the following sections.

\section{Identification of PrkC substrates}

To identify the targets of PrkC in B. anthracis, the lysates of Bas-wt and Bas $\Delta$ prkC were resolved by two-dimensional gradient SDSPAGE. The gels were stained with phosphorylation-specific Pro-Q Diamond stain and the signal was normalized to total protein levels by SYPRO Ruby staining. We selected two types of protein spots in Pro-Q stained gels (Fig. 1c and S1): (1) proteins that are present in both the strains but have higher intensity in Bas-wt as compared with Bas $\Delta p r k C$ (PrkC-influenced), and (2) proteins in Bas-wt that are absent in Bas $\Delta p r k C$ (PrkC-specific). These differentially phosphorylated protein spots were identified by mass spectrometry (Table 1). We could identify 20 different proteins, of which 9 were PrkC-specific while 10 were PrkCinfluenced. Our analysis identified GroEL phosphorylation as a signature of PrkC activity in B. anthracis.

To confirm the substrates of PrkC, we followed two more strategies: immunoprecipitation and phosphoenrichment. Immunoprecipitation was performed with antibodies specific to pSer and pThr using $B$. anthracis whole cell protein lysate. Using this approach, seven prominently phosphorylated proteins were identified by mass spectrometry (Table 2). Phosphoenrichment was performed using lysates of strains Bas-wt and Bas $\triangle p r k C$ (using Qiagen Phosphoenrichment kit). Equal amounts of phosphoenriched proteins from both the samples were analyzed by SDSPAGE to detect the differentially enriched proteins because of variable phosphorylation, followed by mass spectrometry to identify the PrkC phosphorylated proteins (Table 3).

Three substrates identified in screening were independently validated by in vitro phosphorylation assay using recombinant PrkC. Time-dependent phosphorylation kinetics showed optimal time for efficient in vitro phosphorylation (Fig. 2a, b, S2). Ef-Tu that has previously been shown to be phosphorylated by PrkC (and homologs) in B. anthracis, Bacillus subtilis (B. subtilis), and $M$. tuberculosis was used as a positive control to corroborate our results. ${ }^{14,20,21,29}$ The experiment confirmed phosphorylation of SodA2, Ef-G, and GroEL by PrkC (Fig. 2, S3). PrkC-K40M mutant was

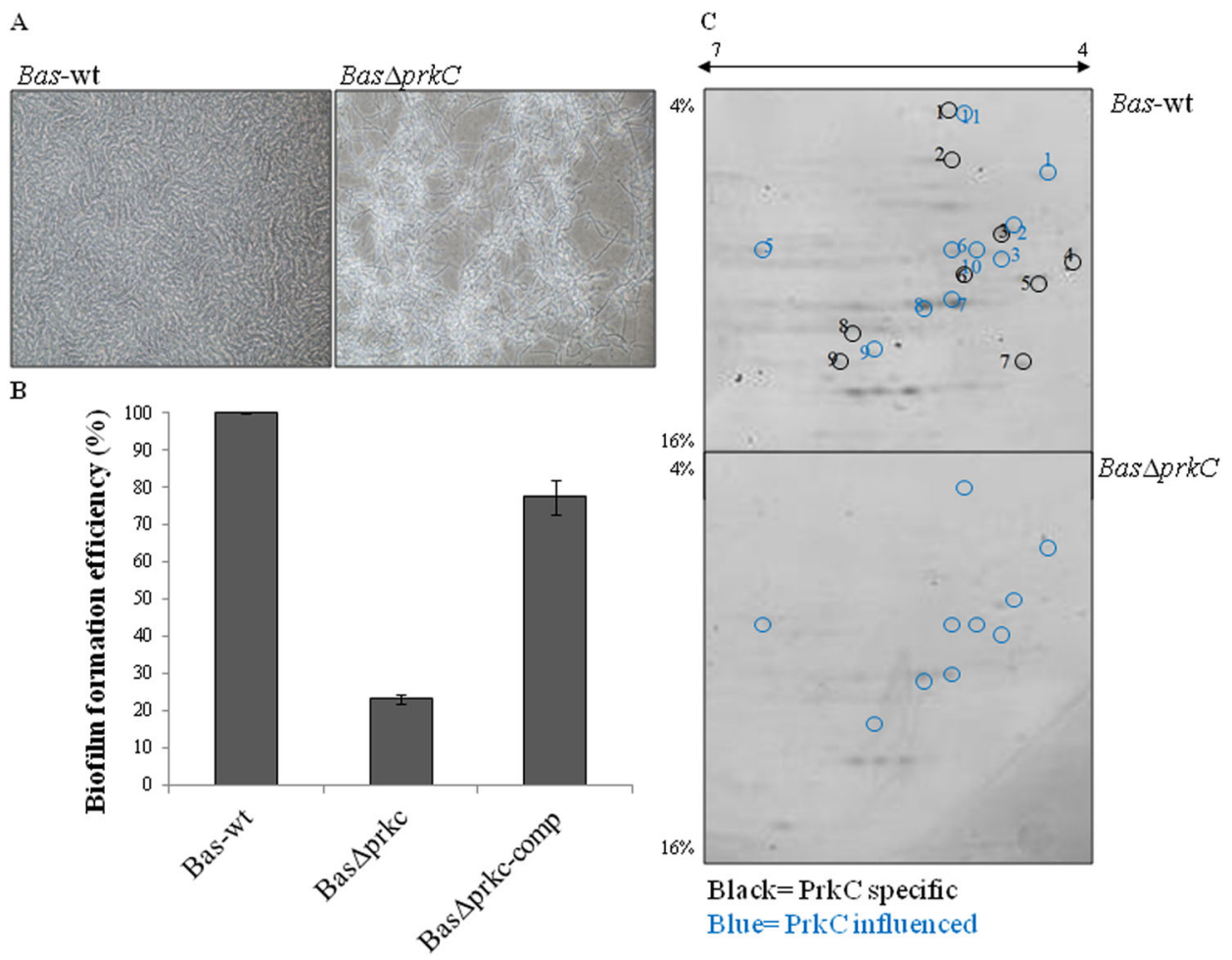

Fig. 1 PrkC-dependent biofilm formation in B. anthracis and identification of in vivo substrates: a Biofilm formation in 3-day static culture of Bas-wt and Bas $\Delta p r k C$ strains after staining with crystal violet as observed under microscope. b Efficiency of biofilm formation in Bas-wt, $B a s \Delta p r k C$, and complemented Bas $\Delta$ prkC strains were calculated by crystal violet assay. Extent of biofilm formation was calculated considering Bas-wt as $100 \%$. The experiment was performed thrice and the error bars indicate the standard error (SE) of three independent values. c Bas-wt and Bas $\Delta$ prkC lysates were separated by two-dimensional PAGE using 4-16\% gradient gels and visualized with Pro-Q diamond phosphospecific staining. These proteins were subsequently analyzed by staining the gels with SyproRuby stain (Fig. S1). The more intense (blue numbered, PrkC-influenced) or unique spots (black numbered, PrkC-specific) in Bas-wt as compared with Bas $\Delta$ prkC were analyzed by mass spectrometry (Table 1). The bands are also encircled with corresponding color 
Table 1. Differentially phosphorylated proteins identified by mass spectrometry

\begin{tabular}{|c|c|c|}
\hline \multicolumn{3}{|l|}{ PrkC-specific } \\
\hline 1 & Bas0107, fusA & Elongation factor-G \\
\hline 3 & Bas0253, groEL & GroEL, $60 \mathrm{kDa}$ chaperone \\
\hline 4 & Bas4937, murB & UDP-N-acetylenolpyruvoylglucosamine reductase \\
\hline 7 & Bas3882 & Pyruvate dehydrogenase E1 component subunit beta \\
\hline 8 & Bas4070 & Leucine dehydrogenase \\
\hline 9 & Bas3677, tsf & Elongation factor-Ts \\
\hline \multicolumn{3}{|c|}{ PrkC-influenced } \\
\hline 1 & Bas4213, dnaK & Molecular chaperone DnaK \\
\hline 5 & Bas0295 & 1-Pyrroline-5-carboxylate dehydrogenase \\
\hline 6 & Bas3392 & DNA topoisomerase IV subunit B, DNA gyrase \\
\hline 7,8 & Bas0108, tuf & Elongation factor-Tu \\
\hline 9 & Bas4297, aspS & Aspartyl-tRNA synthetase \\
\hline 10 & Bas1408, rpsA & $30 S$ ribosomal protein $\mathrm{S} 1$ \\
\hline 11 & Bas3408 & Aconitate hydratase \\
\hline
\end{tabular}

Table 2. List of proteins identified by immunoprecipitation with $a$ pSer/a-pThr antibodies

\begin{tabular}{llcl}
\hline Protein identified & Gene ID & MS score & $\begin{array}{l}\text { Sequence } \\
\text { coverage }\end{array}$ \\
\hline Elongation factor Tu & Bas0108 & 99 & $50.5 \%$ \\
Superoxide dismutase & Bas5300 & 105 & $60.3 \%$ \\
GroEL, 60 kDa chaperone & Bas0253 & 97 & $44.1 \%$ \\
Aldehyde dehydrogenase & Bas3348 & 113 & $54.5 \%$ \\
1-Pyrroline-5-carboxylate & Bas0295 & 197 & $52.0 \%$ \\
dehydrogenase & & & \\
Formate acetyltransferase & Bas0481 & 94 & $42.3 \%$ \\
Alcohol dehydrogenase & Bas2111 & 144 & $59.7 \%$ \\
\hline
\end{tabular}

used as negative control, which is incapable of carrying out phosphorylation. ${ }^{14,15}$ Subsequently, to test the reversibility of phosphorylation, phosphorylated GroEL was incubated with PrpC, which led to its dephosphorylation (Fig. 2d). These results demonstrate that the kinase-phosphatase pair PrkC-PrpC regulates GroEL phosphorylation reversibly. Since the chaperone protein GroEL is involved in biofilm formation in Streptococci and Mycobacteria, ${ }^{30,31}$ and was the only phosphorylated protein identified by all three methods, we investigated the role of PrkC in regulating $B$. anthracis GroEL.

Expression and phosphorylation status of GroEL in B. anthracis To understand the role of GroEL in biofilm formation, we compared its expression profile in Bas-wt and Bas $\Delta p r k C$ cells (grown in liquid media with shaking) with Bas-wt biofilms (grown in static condition), using immunoblotting with anti-GroEL antibodies. There was no change in expression of GroEL in the Bas $\Delta p r k C$ strain as compared with Bas-wt, but the expression increased marginally in biofilm-forming cells, indicating that it might be important under such conditions (Fig. 3a). To understand the in vivo status of GroEL phosphorylation, we overexpressed GroEL in Bas-wt and Bas $\Delta p r k C$. Overexpressed GroEL purified from Bas-wt and Bas $\Delta p r k C$ was subjected to immunoblotting using anti-pThr antibodies and the result shows PrkC-mediated phosphorylation of GroEL in Bas-wt (Fig. 3b). Further to understand the stoichiometry of GroEL phosphorylation, we parsed the phosphorylated and unphosphorylated isoforms. Whole cell protein extracts from Bas-wt and Bas $\triangle p r k C$ strains were resolved by two-dimensional PAGE followed by immunoblotting using anti-GroEL antibody. In Bas $\Delta p r k C$, we observed only one GroEL protein isoform that migrated to an approximate pl of 4.7 (Fig. 3c). However in Bas-wt cells, we identified four isoforms of GroEL, of which one was at pl similar to that in Bas $\Delta p r k C$ strain (i.e., 4.7 ), and the other spots migrated to a lower pl range nearing 4.0 (Fig. $3 \mathrm{C}$ ). Since the phosphorylated species are more acidic in nature, we concluded that the additional GroEL isoforms refer to the phosphorylated species. This indicated the presence of phosphorylated species of GroEL in Bas-wt as compared with single species (unphosphorylated) in Bas $\Delta p r k C$ strain. The stoichiometry of GroEL phosphorylation was subsequently assessed in biofilm-forming cells (Bas-BF). We identified multiple isoforms with lower pl as compared with Bas $\Delta p r k C$ strain and Bas-wt cells (Fig. 3c). These results clearly indicate that GroEL expression and its phosphorylation are associated with biofilm formation, and it is imperative to analyze the underlying regulatory process.

Co-expression of GroEL with PrkC/PrpC and effect of phosphorylation on oligomerization

Since GroEL, a protein-folding chaperone, is essential for major cellular processes and development including biofilm formation in 
Table 3. Phospho-enriched proteins in Bas-wt identified by mass spectrometry

\begin{tabular}{llrr}
\hline Protein identified & Gene ID & MS score & Sequence coverage \\
\hline Leucine dehydrogenase & Bas4070 & 151 & $32 \%$ \\
GroEL, 60 kDa chaperone & Bas0253 & 239 & $45 \%$ \\
Short-chain Enoyl-coA hydratase & Bas4420 & 86 & $33 \%$ \\
Nucleoside diphosphate kinase & Bas1425 & 150 & 160 \\
Respiratory nitrate reductase, alpha subunit & Bas1977 & 213 & $33 \%$ \\
Phosphoglucomutase/Phosphomannomutase family protein & Bas4790 & 118 & $58 \%$ \\
Zinc-containing alcohol dehydrogenase & Bas0641 & 85 & $34 \%$ \\
Hypoxanthine-guanine phosphoribosyltransferase & Bas0063 & 125 & $45 \%$ \\
Hypothetical protein, HTH arsenical resistance operon repressor domain & Bas4146 & & $50 \%$ \\
& & &
\end{tabular}
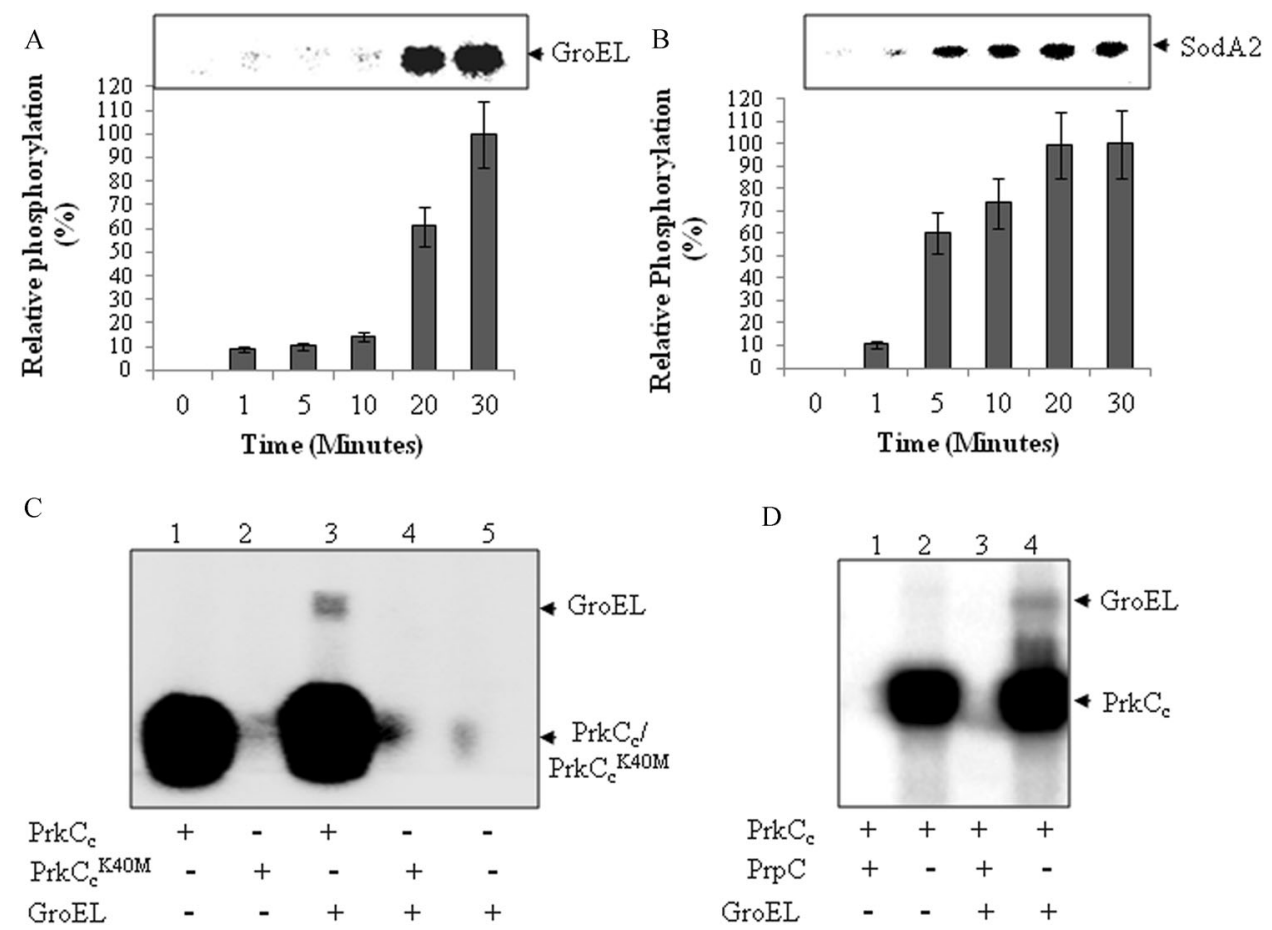

Fig. 2 Time-dependent phosphorylation kinetics of PrkC and reversible phosphorylation of GroEL: To validate GroEL as PrkC substrate (Tables 1, 2, and 3), we used recombinant GroEL $(57.5 \mathrm{kDa})$ for in vitro kinase assays using the kinase domain of PrkC [PrkC $\mathrm{C}_{c} 1-337$ aa, $\sim 40$ $\mathrm{kDa}^{14}$ ]. Autoradiogram shows Time-dependent phosphorylation of GroEL (a) and SodA2 (b), with autophosphorylated kinase (using cold ATP). Phosphorylation intensity at 30 min time point was taken as $100 \%$ and relative phosphorylation was calculated followed by normalization with the protein amount (Fig. S2). The corresponding autoradiogram is shown above the bar graph. The assay was performed twice and intensities were calculated three times each. Error bars represent SE of six independent calculations. c Autoradiogram showing in vitro phosphorylation of GroEL. PrkC catalytic domain (PrkC $1 \mu \mathrm{g}$ ) was used for phosphorylation (for $30 \mathrm{~min}$ ) of purified GroEL (5 $\mu \mathrm{g}$ ) and the kinase dead mutant ( PrkC $\left._{c}-\mathrm{K} 40 \mathrm{M}\right)$ was used as a negative control. No phosphorylation was observed in the control reactions when the kinase inactive mutant PrkC $\mathrm{C}_{\mathrm{c}}-\mathrm{K} 40 \mathrm{M}$ was used. ${ }^{14} \mathbf{d}$ Autoradiogram showing in vitro dephosphorylation of PrkC-phosphorylated GroEL by Ser/Thr phosphatase PrpC $(1 \mu \mathrm{g})$

some bacteria, ${ }^{31-33}$ we wanted to further understand the molecular implications of PrkC-mediated GroEL phosphorylation. GroEL was expressed in the presence of either PrkC or PrpC in the surrogate host Escherichia coli (E. coli). The phosphorylation was confirmed by metabolic labeling with $\left[{ }^{32} \mathrm{P}\right]$ orthophosphoric acid (Fig. 4a) and Pro-Q Diamond staining (Fig. 4b). PrkC readily phosphorylated GroEL (GroEL-P), whereas no phosphorylation was observed in the presence of the corresponding phosphatase PrpC (GroEL-UP) (Fig. 4a, b). The stoichiometry of GroEL-P and GroEL-UP was analyzed by two-dimensional PAGE-based separation followed by immunoblotting with anti-GroEL antibodies. We found multiple isoforms generated after phosphorylation in GroEL-P and separated on the basis of their isoelectric points (Fig. 4c). In contrast, a single species of GroEL-UP was observed, indicating the lack of phosphorylation. These results are in agreement with above-mentioned status of GroEL phosphorylation during biofilm formation (Fig. 3b).

To understand the impact of GroEL phosphorylation, we analyzed the chaperonin function on the basis of structural organization of GroEL. The chaperone complex is formed by specialized tetradecameric GroEL rings that work in conjunction with a heptameric GroES cap. ${ }^{34}$ GroEL monomeric forms have negligible folding activity in vitro and the oligomeric structure of GroEL/GroES is required for biologically significant chaperonin 
A

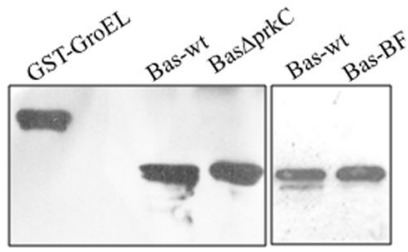

B

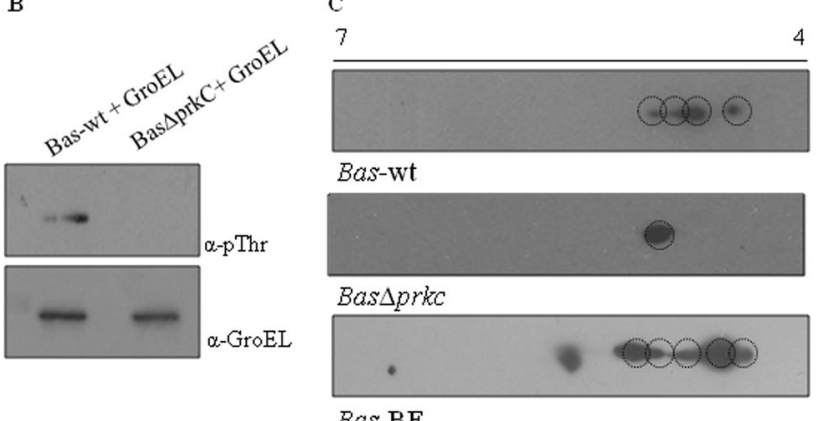

Fig. 3 GroEL expression and phosphorylation in B. anthracis: a Immunoblots show the expression of GroEL in B. anthracis cell lysates. Equal amounts of lysates ( $5 \mu \mathrm{g}$ each) were used and probed with anti-GroEL antibodies. Purified GST-GroEL (0.5 $\mu \mathrm{g}$ ) was taken as the positive control. Left panel shows the comparative expression of GroEL in Bas-wt and Bas $\Delta$ prkC. Right panel shows the comparative expression of GroEL in Baswt and BasBF. b groEL was overexpressed and purified from B. anthracis strain, followed by immunoblotting with anti-pThr antibody. GroEL was phosphorylated in Bas-wt whereas no phosphorylation was observed with GroEL purified from Bas $\Delta p r k C$. c Two-dimensionally separated cell lysates of $B$. anthracis were probed with anti-GroEL antibodies to assess the stoichiometry of phosphorylation of native GroEL. Multiple species were observed in Bas-wt as compared with Bas $\Delta p r k C$, showing the in vivo phosphorylation of GroEL specifically by PrkC. GroEL was hyperphosphorylated during biofilm formation (BasBF) as observed by the increased number of spots (third panel)

function. $^{32}$ We performed size exclusion chromatography of GroEL-P and GroEL-UP (using Superose 6 column) to determine GroEL complexes in both forms. Multimeric forms of GroEL-UP eluted as three peaks at $8.5,13.5$, and $15 \mathrm{ml}$, corresponding to tetradecamer (14-mer), heptamer (7-mer), and dimer (2-mer), respectively (Fig. 4d). Interestingly, maximum proportion of GroEL$P$ was found to be in the tetradecamer form. This indicated that phosphorylation of GroEL increases intermolecular interactions resulting in the formation of active tetradecamers. This observation also led us to speculate that PrkC-mediated phosphorylation might occur at the oligomerization interface of GroEL (equatorial domain).

Effect of phosphorylation on GroES:GroEL interaction The activity of GroEL is dependent on the successful interaction with the co-chaperone, GroES. To evaluate the effect of GroEL phosphorylation on its interaction with GroES, we utilized proteinase $\mathrm{K}$ resistance assay. In the absence of GroES, proteinase $\mathrm{K}$ preferentially cleaves GroEL at the accessible C-terminal region, resulting in a truncated protein of $\sim 52 \mathrm{kDa}$. The compact doublering cylindrical GroEL structure becomes more stable in the presence of GroES and is therefore protected from proteinase Kmediated proteolysis. ${ }^{35}$ We tested GroES-mediated protection of GroEL-P and GroEL-UP, and measured the uncleaved GroEL fraction. We observed that GroES protected the phosphorylated form of GroEL (53\%) more than the unphosphorylated form (36\%) from the proteinase $\mathrm{K}$ cleavage (Fig. $4 \mathrm{e}$ ). This indicate that GroES: GroEL-P interaction is stronger than GroES:GroEL-UP, and therefore GroEL-P represents the active form of protein.

Identification of phosphorylation sites in GroEL and their structural arrangement

Presence of multiple isoforms on two-dimensional PAGE led us to hypothesize that GroEL-P is phosphorylated on more than one amino acid residues (Fig. 3c). To validate this hypothesis, GroEL-P and GroEL-UP were subjected to mass spectrometry. The analysis identified six phosphorylated threonine residues in GroEL-P-Thr21, Thr132, Thr172, Thr184, Thr328, and Thr329 (Fig. 5). Mass spectrometry did not identify any phosphorylated residue in GroEL-UP, indicating the specificity of PrkC-mediated GroEL phosphorylation. To study the contribution of individual sites in GroEL phosphorylation, the non-phosphorylatable mutants were generated as-GroEL-T21A, GroEL-T132A, GroEL-T172A, GroELT184A, GroEL-T328A, GroEL-T329A, and the double mutant GroEL-
T328/329A. The phosphorylation of GroEL was compared with each of these mutants. As shown in Fig. 6a, GroEL-T21A, GroELT132A, and GroEL-T328/329A showed maximum loss in phosphorylation compared with GroEL. These results suggest that Thr21, Thr132 and either of Thr328 or Thr329 are the major phosphorylation sites in GroEL.

Subsequently, we assessed the arrangement of phosphorylation sites in GroEL structure. Protein sequence of GroEL is highly conserved, with $64 \%$ identity to E. coli GroEL. On the basis of this similarity, we generated the three-dimensional structure of $B$. anthracis GroEL through homology modeling using Modeller v9.13 (Fig. 6b). The structure shows that two of the phosphorylation sites Thr328 and Thr329 lie in the apical domain of GroEL, Thr21, and Thr132 are present in the equatorial domain, and Thr174 and Thr182 are present in the intermediate domain (Fig. 6c). Since oligomerization of $B$. anthracis GroEL is influenced by phosphorylation (Fig. 4d), we tried to understand which residues play an important role in this regulation and thereby overall activity of $B$. anthracis GroEL.

Complementation of the groEL44 allele in E. coli

To establish that phosphorylation is essential for GroEL activity in vivo, effect of GroEL phosphorylation was subsequently tested for complementation of $E$. coli GroEL. E. coli SV2 strain harbors a temperature-sensitive groEL44 allele and cannot be grown at $42{ }^{\circ} \mathrm{C}$ unless expressing a functional GroEL. ${ }^{36}$ To understand the functionality of GroEL, we expressed B. anthracis GroEL along with its cognate GroES in E. coli SV2 at permissive $\left(30^{\circ} \mathrm{C}\right)$ and restrictive $\left(42^{\circ} \mathrm{C}\right)$ temperatures. The expression of $B$. anthracis GroEL and GroES did not lead to E. coli SV2 growth at $42^{\circ} \mathrm{C}$ (Fig. 7a). Similarly, there was no growth when nonphosphorylatable mutants of GroEL ( $p$ Thr to Ala) were expressed (data not shown). Considering the fact that B. anthracis GroEL is active in the phosphorylated form, we expressed the phosphomimetic mutants (pThr to Glu) of selected sites that showed major loss of phosphorylation in GroEL (GroEL-Thr132, Thr182, and Thr329, Fig. 7a) in E. coli SV2. Interestingly, the cells expressing phospho-mimetic mutants were able to survive at restrictive temperature, whereas the wild-type GroEL could not survive (Fig. 7a). Of all the mutants, B. anthracis GroEL ${ }^{\mathrm{T} 329 \mathrm{E}}$ was most functional at the $42{ }^{\circ} \mathrm{C}$ and was able to complement groEL44 more successfully. 
A

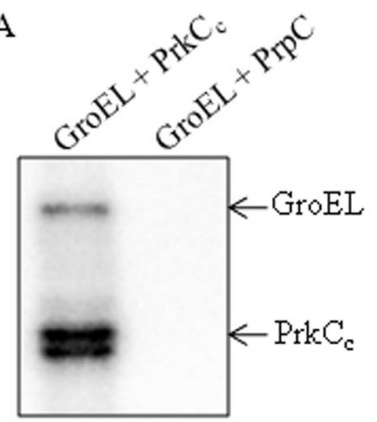

B

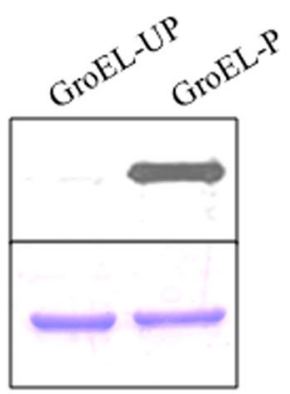

C 7

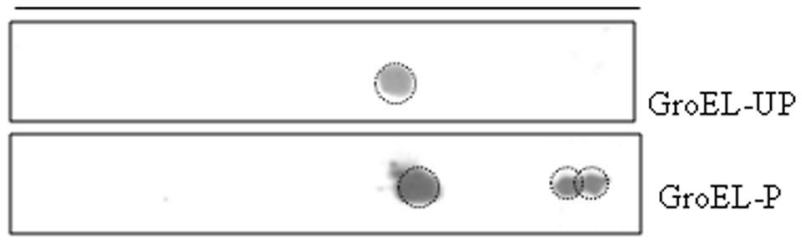

$\mathrm{D}$
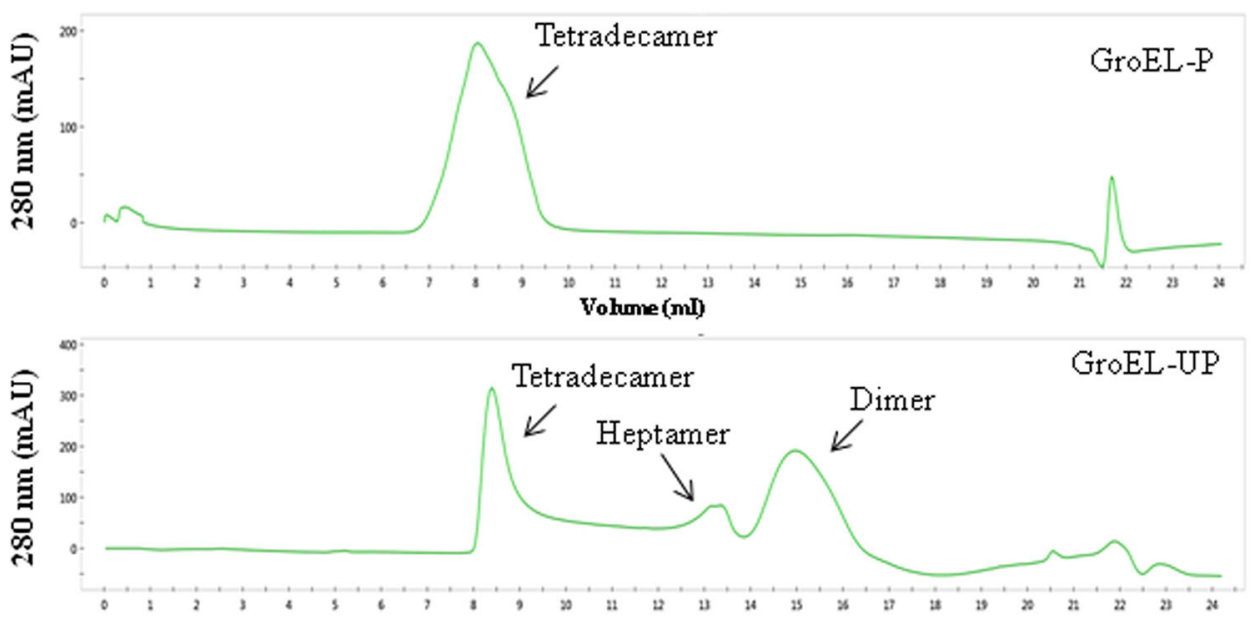

Vohme (mI)

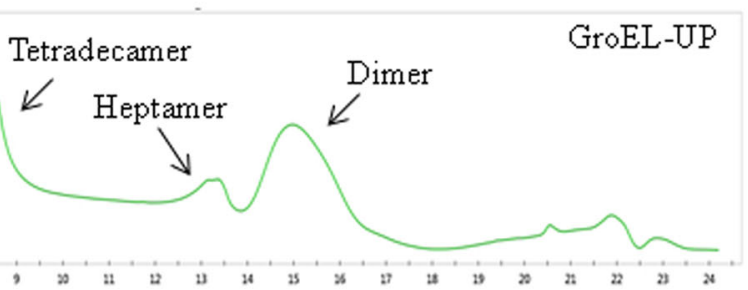

Vohume(mI)
E

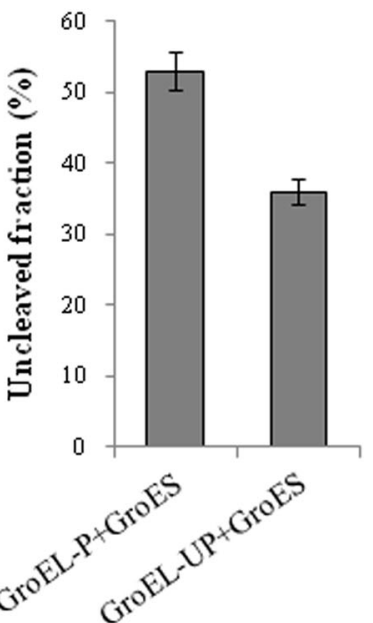

Fig. 4 Co-expression of GroEL with PrkC and PrpC in E. coli and effect of phosphorylation on GroEL: a E. coli BL21 cells overexpressing His ${ }_{6}^{-}$ GroEL with either PrkC $C_{c}$ or PrpC were metabolically labeled using ${ }^{32}$ P] orthophosphoric acid. As shown in the autoradiogram, GroEL is phosphorylated in the presence of PrkC. Due to its affinity for GroEL, PrkC ${ }_{c}$ was also co-precipitated. b Purified His ${ }_{6}-G$ roEL co-expressed with either PrkC $C_{c}$ or PrpC were resolved by SDS-PAGE. The gel was stained with Pro-Q stain and analyzed by Typhoon imager. GroEL was

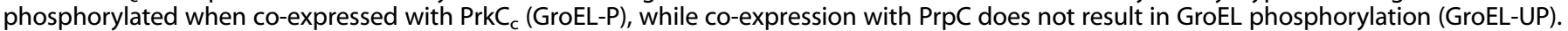
c To understand the stoichiometry of phosphorylation of GroEL-P and GroEL-UP, the purified proteins (1 $\mu \mathrm{g}$ each) were separated by twodimensional PAGE (pl range 4-7). The gels were immunoblotted on nitrocellulose membrane and developed using anti-GroEL antibodies. The images show respective autoradiograms of GroEL-UP (upper panel) and GroEL-P (lower panel). Multiple species of GroEL-P were observed indicating different levels of phosphorylation. d Gel filtration of purified GroEL-P and GroEL-UP. The graphs show presence of higher ratio of tetradecameric species in GroEL-P (upper panel) as compared with GroEL-UP (lower panel), which showed majority of dimer and heptamer. e Interaction of GroES with GroEL-P and GroEL-UP were analyzed by proteinase K-mediated partial cleavage. As compared with GroEL-UP: GroES, the phosphorylated GroEL-P:GroES complex was much more protected from protease cleavage indicating a stronger interaction. The experiment was performed thrice and the error bars show the SE of three independent readings

Phenotypic complementation of PrkC by GroEL

Our results establish that GroEL is an important substrate of PrkC and its structural stabilization and activity is dependent on PrkCmediated phosphorylation. Since prkC deletion caused loss in biofilm formation (Fig. 1), and if it is mediated by the downstream substrate GroEL, the loss should get complemented by increasing GroEL concentration. To test this hypothesis, GroEL was overexpressed in Bas $\Delta p r k C$ strain and its effect was analyzed on biofilm formation. Expressing GroEL at higher concentration led to a partial resumption of biofilm formation in Bas $\Delta p r k C$ strain (Fig. 7b). This result showed that GroEL is required for biofilm formation in B. anthracis and its overexpression can compensate for the defect in PrkC.

To further understand the role of phosphorylated residues in GroEL on biofilm formation, we overexpressed each nonphosphorylatable GroEL mutant, GroEL-T21A, GroEL-T132A, and GroEL-T329A, in Bas $\Delta p r k C$ and compared the biofilm formation with Bas-wt and Bas $\triangle p r k C+$ groEL strains (Fig. 7c). As evident from the Fig. 7c, Bas $\Delta p r k C$ strain did not form biofilm when GroEL phospho-ablative mutants were overexpressed, and thus were not able to compensate for the loss of PrkC as compared with native GroEL. This data indicated that Thr21, Thr132, and Thr329 are critical for the activity of GroEL, and any perturbation of these residues may influence GroEL activity, and consequently the biofilm-forming ability of $B$. anthracis.

Phosphorylation of GroEL in other bacteria

PrkC homologs phosphorylate multiple substrates and have a role in virulence and bacterial development. ${ }^{14}, 15,19,21,22,27,37-40$ To test the dependency of GroEL phosphorylation on PrkC in $B$. subtilis, a close relative of $B$. anthracis, we compared the phosphorylation status of GroEL by protein isoform analysis. On comparing the $B$. subtilis wild-type, $B$. subtilis $\Delta p r k C$, and $B$. subtilis prkC complemented strains, we observed that only a single species of GroEL was present in the $B$. subtilis $\Delta$ prkC strain whereas multiple isoforms were observed in the presence of PrkC (Fig. 8b). Thus, as in B. anthracis, GroEL is specifically phosphorylated by PrkC in B. subtilis. Since PrkC is a conserved Ser/Thr kinase that can get activated by sensing shared environmental cues, so bacteria may share common regulatory strategies involving PrkC and 
(R)GVDp TLANAVK(V), Thr21

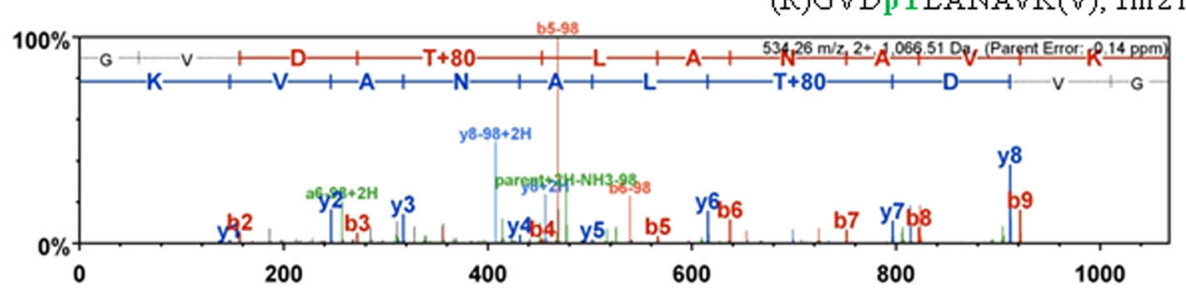

(K)AVVAAVEELKp TISKPIEGK(S), Thr 132

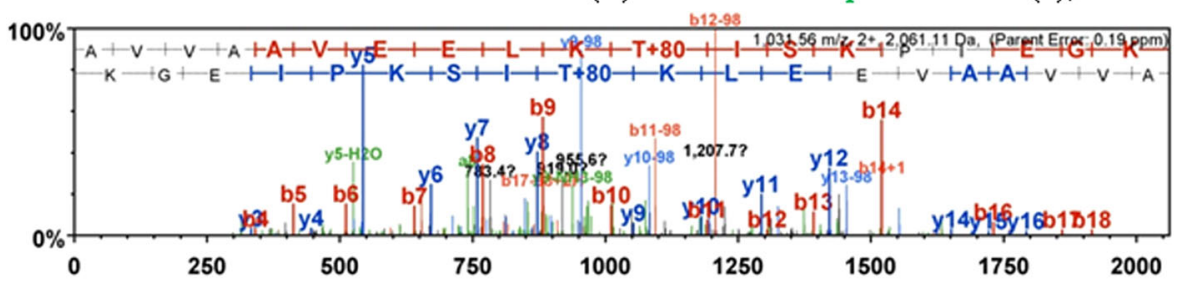

(R)VGNDGVIpTLEESK(G), Thr 174

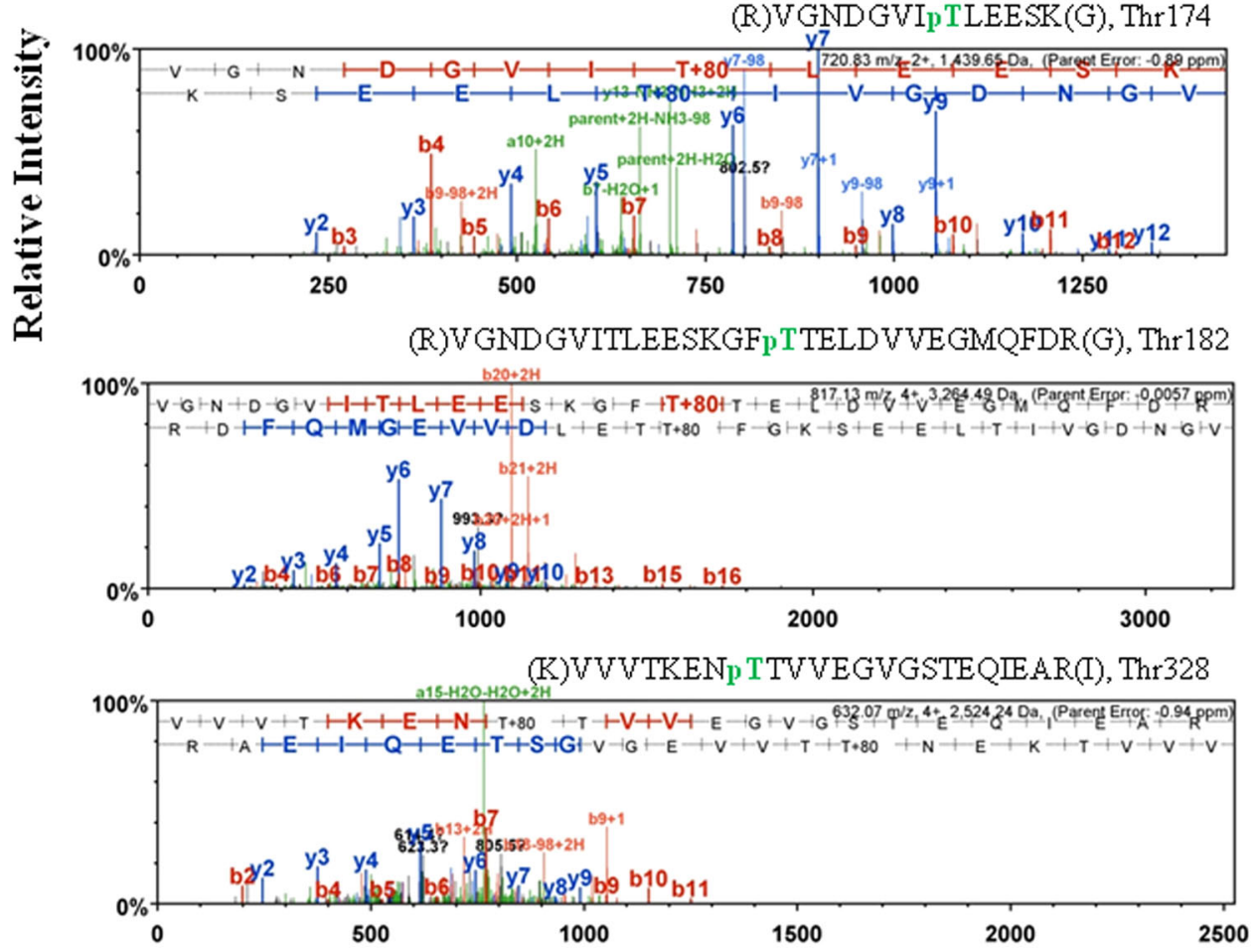

(K)VVVTKENTp TVVEGVGSTEQIEAR(I), Thr329

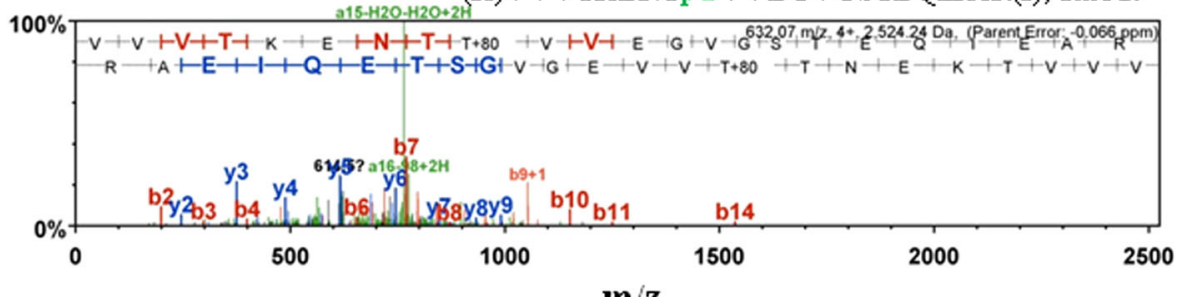

$\mathbf{m} / \mathbf{z}$

Fig. 5 Phosphorylation sites of GroEL: mass spectrometry spectra showing the phosphorylation sites of GroEL. The spectra were displayed using the Scaffold software and corresponding trypsinized peptides are shown. Phosphorylated threonine residues within the identified peptides are marked in green

GroEL. To confirm this, we selected five diverse bacterial species that encode a chaperone with close homology to $B$. anthracis GroEL (Supplementary File, multiple sequence alignment). We prepared whole cell lysates of bacteria-B. subtilis, Staphylococcus aureus (S. aureus), Streptococcus agalactiae (S. agalactiae), Pseudomonas aeruginosa ( $P$. aeruginosa), and Mycobacterium smegmatis (M. smegmatis)-and performed two-dimensional SDS-PAGE followed by immunoblotting with $B$. anthracis GroEL antibodies 

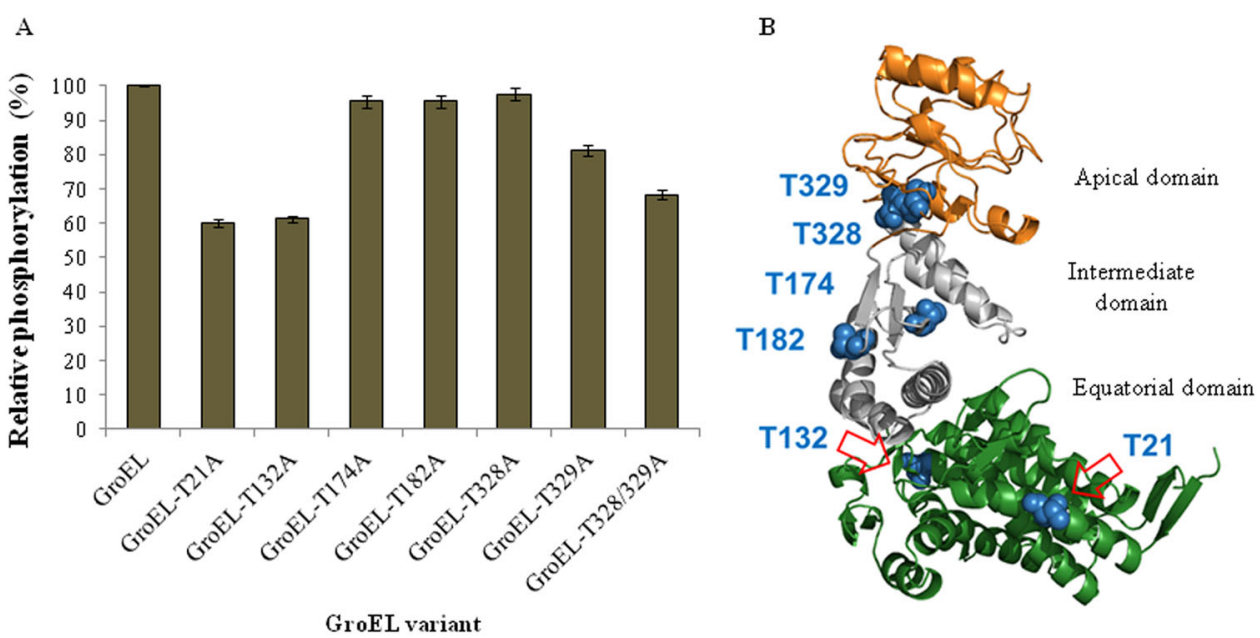

C

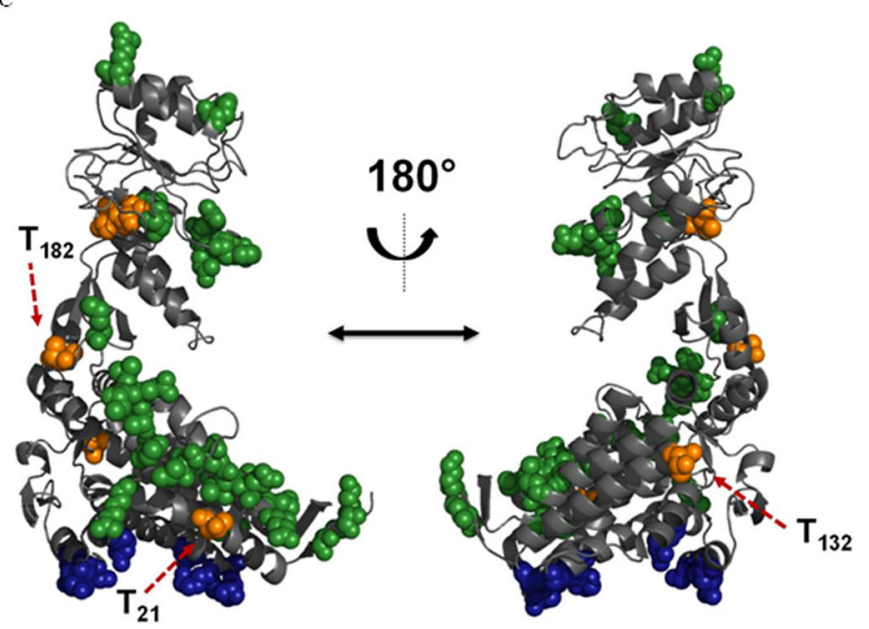

Fig. 6 Structural localization of GroEL phosphorylation sites: a To understand the contribution of individual phosphorylation sites in GroEL, non-phosphorylatable mutants (Thr to Ala) were generated and were used for in vitro kinase assays with PrkC $\mathrm{C}_{\mathrm{c}}$. The band intensities on autoradiogram were estimated by QuantityOne (BioRad). Phosphorylation signal on native GroEL band was taken as $100 \%$ and relative phosphorylation was calculated in the remaining samples. The experiment was performed thrice and the error bars indicate the SE of three independent values. As clearly evident, major loss in phosphorylation was observed in GroEL-T21A, GroEL-T132A, and GroEL-T328/329A. b The structural model of GroEL was generated by Modeller v9.13 using the co-ordinates from E. coli GroEL structure (PDB code: 1AON chain A). The diagrammatic representation depicts three distinct regions in GroEL: apical (gold), intermediate (silver), and equatorial (green). The figures were generated in Pymol 1.3. Phosphorylated threonine residues are indicated as spheres in blue. The six phosphorylation sites have been marked in the respective regions. c Molecular model depicting the three-dimensional structure of B. anthracis GroEL monomer. The phosphothreonine residues (broken red arrows) possibly involved in multimerization have been marked. Molecular visualizations were performed using the Pymol Molecular Graphics System v1.3

and M. tuberculosis GroEL2 antibodies. In this experiment, we detected multiple isoforms of GroEL in all these bacteria, possibly indicating the phosphorylated species (Fig. 8a). Thus, GroEL phosphorylation seems to be a conserved phenomenon; however, the sites of phosphorylation and the mechanistic details of such regulation still needs to be defined.

\section{DISCUSSION}

Many species of pathogenic bacteria are difficult to eradicate due to formation of resistant biofilms and spores. PrkC, a sensory STPK, and its homologs control the bacterial cell fate being the key regulator of cellular development and physiology. In B. subtilis, PrkC regulates spore formation as well as germination and biofilm formation, ${ }^{41}$ although the role of other signaling modules has also been shown under certain conditions. ${ }^{42,}{ }^{43}$ Similar roles of PrkC in biofilm formation have been indicated in Staphylococcal sp. pathogens, ${ }^{39,} 44$ but the molecular mechanism remains unknown. PrkC also affects the pathogenicity of Streptococcus mutans through regulation of multiple processes, such as genetic transformability, cell shape and division, growth and stress response. ${ }^{37,45}$ In this study, we have shown that PrkC plays a critical role in B. anthracis biofilm formation, with GroEL as the primary mediator. GroEL is an essential chaperone present in diverse bacteria that forms nanocages and provide central compartment to prevent aggregation of unfolded proteins. ${ }^{46}$ Employing biochemical and proteomic strategies, we found that GroEL is one of the most consistent substrate of $B$. anthracis PrkC, and this phosphorylation is conserved among other bacterial species.

B. anthracis GroEL is highly immunogenic and generates stronger immune response. ${ }^{47}$ Mice pre-injected with GroEL are protected against anthrax infection, indicating that GroEL might be important for pathogenesis. ${ }^{48}$ GroEL is co-transcribed with the co-chaperone GroES and the GroEL-GroES complex mediates appropriate protein folding. GroEL forms homo-heptamers and two such heptamers join to form a tetradecameric ring-like 

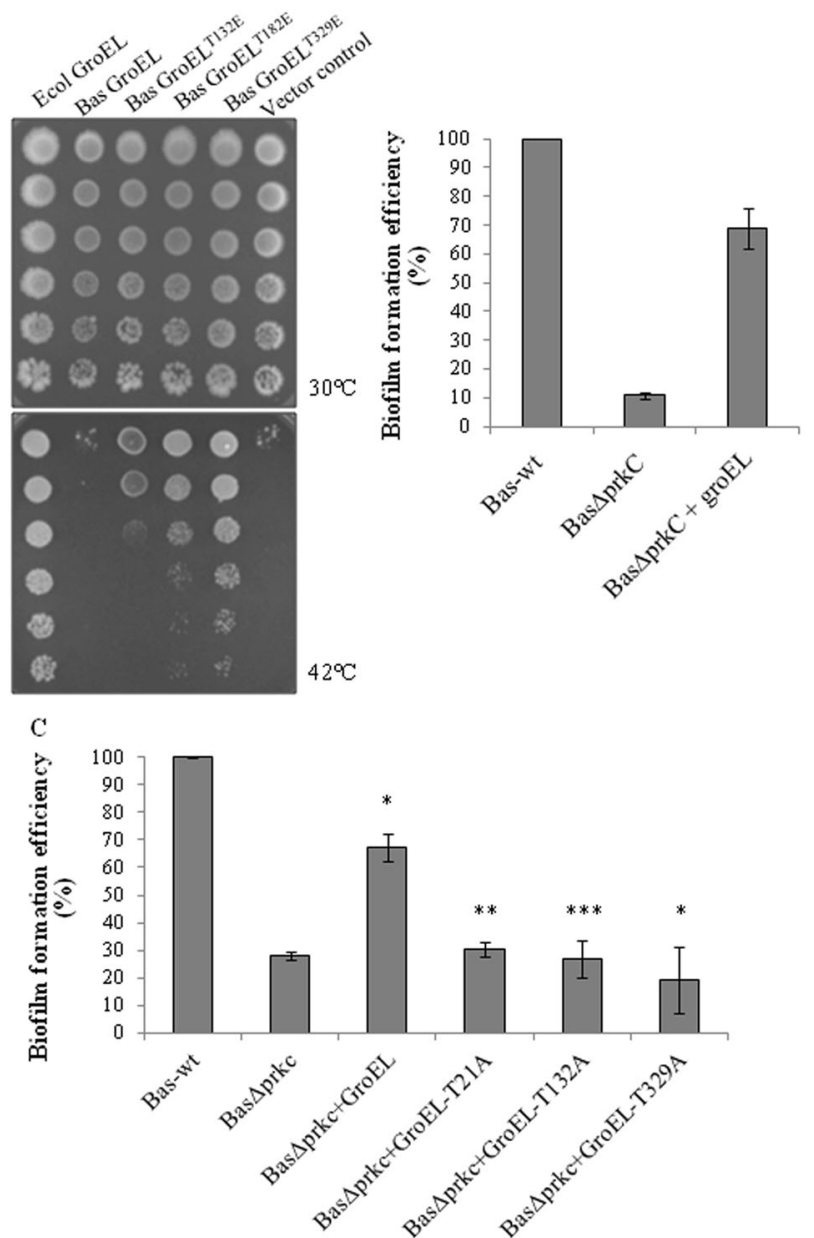

Fig. 7 Role of GroEL phosphorylation sites: a B. anthracis GroEL and GroES were expressed in E. coli SV2 temperature sensitive strain and spotting was performed. To assay the effect of GroEL phosphorylation, specific phospho-mimetic mutants were also expressed at $30^{\circ} \mathrm{C}$ (upper panel) and $42^{\circ} \mathrm{C}$ (lower panel). All the strains grew similarly at permissive temperature $\left(30^{\circ} \mathrm{C}\right)$ but not at $42{ }^{\circ} \mathrm{C}$. As compared with native GroEL, cells expressing phospho-mimetic mutants were able to grow at $42{ }^{\circ} \mathrm{C}$. The images show serially diluted cells from top to bottom in both the panels. b The biofilm formation efficiencies of Bas-wt and Bas $\Delta$ prkC strains were compared with Bas $\Delta$ prkC cells overexpressing GroEL and calculated by crystal violet assay. As observed before (Fig. 1b), deletion of prkC led to loss of biofilm formation, which was recovered after overexpression of GroEL. Biofilm formation in Bas-wt was taken as $100 \%$ and relative efficiency was calculated. The experiment was performed thrice and the error bars indicate the SE of three independent values. c The biofilm formation efficiencies of Bas-wt and Bas $\Delta p r k C$ strains were compared with Bas $\Delta p r k C$ cells overexpressing either native GroEL or its phosphorylation site mutant (GroEL-T21A, GroEL-T132A, and GroEL-T329A), using crystal violet assay. The experiment was performed four times and the error bars show SE of four independent biological replicates. The $p$-value was calculated using two-tailed $t$-test, as shown by asterisk $\left({ }^{*} p<0.05,{ }^{* *} p \leq 0.005,{ }^{* * *} p \leq\right.$ $0.001)$

structure, which is assisted by a GroES heptameric cap. ${ }^{32}$ This whole complex mediates folding of unfolded or incorrectly folded peptides, resulting in a correctly folded and active protein. GroEL oligomerization is important for its protein-folding activity and possibly pathogenesis. ${ }^{36}$ Specifically, previous studies in $M$. tuberculosis and E. coli have shown that GroEL phosphorylation induces its multimerization, making it an active chaperone
A

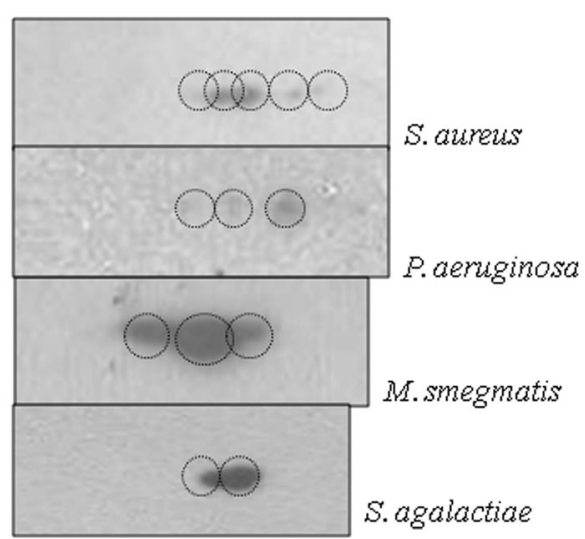

B

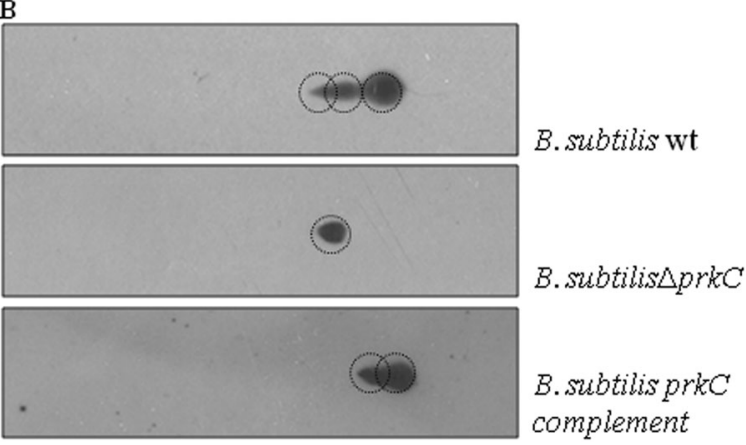

Fig. 8 Conservation of GroEL phosphorylation in multiple bacteria: a The phosphorylation status of GroEL was studied in different bacterial species: S. aureus, P. aeruginosa, M. smegmatis, and S. agalactiae. Owing to its highly conserved sequence (Supplementary File), the cell lysates were probed with $B$. anthracis GroEL antibody, except $M$. smegmatis, which was probed with mycobacterial GroEL antibody. In all the strains studied, GroEL was separated into multiple isoforms, indicating the conservation of phosphorylation. $\mathbf{b}$ The specific phosphorylation of GroEL by PrkC was also studied in $B$. subtilis by using the prkC deletion strain and its complement. Three species of GroEL were observed in B. subtilis wild-type strain (first panel), which were lost in $B$. subtilis $\Delta$ prkC (second panel) and recovered by complementation of prkC (third panel)

complex. $^{36,} 49,50$ We also found that GroEL-P is present in tetradecamer form as compared with unphosphorylated species. In addition, our results show that phosphorylated GroEL efficiently interacts with GroES, thus having a higher tendency to constitute an active complex. This indicates that phosphorylation facilitates the formation of active GroEL-GroES complex in B. anthracis.

The expression of GroEL is induced during stress conditions, such as high temperature, sporulation, or biofilm formation, indicating that GroEL is required for natural stress response of the cell. $^{31,51,52}$ Furthermore, B. anthracis GroEL was found to be phosphorylated in biofilm-forming cells, indicating the necessity of the active protein for biofilm formation. Under these conditions, GroEL might promote the repair of damaged proteins or facilitate the folding of other overexpressed proteins. In addition, GroEL itself forms amyloid-like fibrils $5^{53}$ and it would be interesting if it has any direct physical role in biofilm organization. Although, GroEL is known to be involved in biofilm formation of a number of bacteria such as Haemophilus influenzae, Campylobacter jejuni, S. mutans, and mycobacteria, ${ }^{30,31,54-56}$ we, however, cannot rule out the possible existence of other PrkC substrate proteins that can influence the biofilm formation in co-operation with GroEL. PrkC and its homologs are shown to regulate multiple pathways by phosphorylation of specific proteins and it is possible that defect in biofilm formation is due to GroEL. Deletion of prkC results 
in unphosphorylated GroEL, which is less active, ultimately causing loss of biofilm formation in $B$. anthracis. Complementing the prkC deletion strain with GroEL partially regained its ability to form biofilms, indicating that an excess of unphosphorylated GroEL may partially supplement for phosphorylated active GroEL. To further confirm this phenomenon, we also complemented the prkC deletion strain with GroEL phosphorylation site mutants that were found to be inactive in $E$. coli SV2 complementation experiments. These mutants were not able to retrieve the biofilm formation in Bas $\Delta p r k C$ strain, thus reaffirming our results.

Initiation of bacterial biofilm requires molecular factors for attachment and accumulation. In this complex, multifactorial process, both protein and DNA form biofilm matrix. The composition of the protein includes surface proteins and adhesins that form amyloid fibers. GroEL is often characterized as cell surface protein in gram-positive bacteria that overexpresses during biofilm formation. ${ }^{57}$ In fact $B$. anthracis GroEL has been reported to be present on the exosporium, cell surface, and secretome. ${ }^{48}$ GroEL is also known to bind to plasminogen and help in evading innate immune response. ${ }^{58}$ Opsonization of cell surface GroEL in B. anthracis leads to immunomodulation and protection in mice. ${ }^{48}$ This is the first study that connects the role of PrkC-mediated biofilm formation with GroEL phosphorylation. Being an abundant surface and secreted protein, GroEL nanocages can be a part of biofilm matrix or may aid in preventing proteostatis and aggregation. ${ }^{46}$ Future studies will be needed to define role of GroEL in biofilm formation and cell surface attachment in $B$. anthracis.

\section{METHODS}

Bacterial strains and growth conditions

E. coli strain DH5a (Novagen) was used for cloning and BL21 (DE3) (Stratagene) was used for the expression of recombinant proteins. E. coli cells were grown as described before. ${ }^{13,21}$ B. anthracis Sterne strain (wildtype Bas-wt and Bas $\Delta p r k C^{17}$ ), and B. subtilis were grown in LB broth at $37^{\circ} \mathrm{C}$ with shaking at $200 \mathrm{rpm}$. For solid media, LB-Agar was used for both $E$. coli and B. anthracis. S. agalactiae was grown in BHI broth and agar at $37^{\circ} \mathrm{C}$. S. aureus (ATCC 29213) and P. aeruginosa (ATCC 25668) strains ${ }^{59}$ were maintained on Mueller Hinton broth and agar (Difco, Franklin Lakes, NJ, USA). M. smegmatis was grown in standard culture medium as described before. $^{13,21,17,60}$

Biofilm formation in B. anthracis and crystal violet assay

B. anthracis Sterne strains (Bas-wt, Bas $\triangle p r k C$, Bas-prkC-comp, Bas $\triangle p r k C+g r o E L, \quad B a s \triangle p r k C+g r o E L-T 21 A, \quad B a s \triangle p r k C+g r o E L-T 132 A$, and Bas $\triangle p r k C+g r o E L-T 329 A)$ were grown until late log phase and secondary cultures $(0.01 \%)$ were inoculated in 6 -well plates containing 5 $\mathrm{ml} \mathrm{LB}$ media. The plates were incubated without shaking at $37^{\circ} \mathrm{C}$ for $72 \mathrm{~h}$ and biofilms were observed. For quantitation of biofilms, crystal violet assay was performed as described before. ${ }^{61}$ Microscopic images were taken by an inverted microscope (Nikon Eclipse Ti, Nikon, Tokyo, Japan).

\section{B. anthracis lysate preparation}

Cells were harvested from $50 \mathrm{ml}$ logarithmic phase bacterial culture and washed twice with $1 \times$ PBS. Cells were resuspended in $5 \mathrm{ml}$ of lysis buffer (PBS 1×, protease inhibitor cocktail [Roche], $1 \mathrm{mM}$ PMSF, phosphatase inhibitor cocktail [Pierce], $1 \mathrm{mg} / \mathrm{ml}$ lysozyme, and $1 \mathrm{mM} \mathrm{NaF}$ ) and sonicated for $10 \mathrm{~min}$. The lysates were clarified and protein concentration was estimated by Bradford assay.

Immunoprecipitation of phosphorylated proteins in $B$. anthracis B. anthracis Sterne strain grown to an $\mathrm{OD}_{600}$ of $\sim 1.0$ in $\mathrm{LB}$ broth was harvested and suspended in lysis buffer containing $50 \mathrm{mM}$ Tris- $\mathrm{HCl}[\mathrm{pH}$ 7.5], $10 \%$ glycerol, $0.1 \%$ Triton X-100, $1 \times$ Protease inhibitor, and $1 \mathrm{mM}$ PMSF and $50 \mathrm{ng} / \mu \mathrm{l}$ lysozyme. The suspended cells were incubated at $37^{\circ} \mathrm{C}$ for $30 \mathrm{~min}$ and sonicated for $5 \mathrm{~min}$. After sonication, the lysate was centrifuged at $15,000 \mathrm{xg}$ for $30 \mathrm{~min}$ and the supernatant (containing $10 \mathrm{mg}$ protein) was incubated overnight at $4{ }^{\circ} \mathrm{C}$ with Protein A-Sepharose
(Invitrogen, India) linked to either a-pSer or a-pThr antibodies. Immunoprecipitates were washed several times with $1 \%$ Triton X-100 in $10 \mathrm{mM}$ Tris-Cl $[\mathrm{pH}$ 7.5] and the protein-antibody complex was eluted using Glycine elution buffer [pH 2.0]. The eluted immuno complexes were resuspended in $1 \times$ SDS sample buffer, resolved on SDS-PAGE, and analyzed by mass spectrometric analysis (UDSC, New Delhi) after staining with Coomassie Brilliant Blue R-250.

\section{Phosphoenrichment}

$400 \mu \mathrm{g}$ of cell lysates (Bas-wt and Bas $\Delta p r k C$ ) were phospho-enriched by Phospho-Protein purification kit (Pierce), according to the manufacturer's instructions. The enriched proteins were concentrated and equal amounts were resolved on SDS-PAGE. Differentially enriched proteins were identified by mass spectrometry.

\section{Identification of phosphorylation sites}

To detect the phosphorylated proteins and peptides, the manually picked gel pieces were trypsinized and prepared for mass spectrometric analysis (Supplementary File). ${ }^{62}$

Cloning and mutagenesis of $B$. anthracis genes and complementation in B. anthracis Sterne strain

Gene cloning and site directed mutagenesis was done using standard molecular biology procedures as described before ${ }^{14,22}$ and B. anthracis Sterne strain genomic DNA. The clones were confirmed with restriction digestion and DNA sequencing (Invitrogen). Site-directed mutagenesis was carried out using QuikChange II XL Site-Directed Mutagenesis Kit (Stratagene) using GroEL clones as templates. The details of primers and plasmids are provided in Table S1.

Genes encoding for PrkC (1-657 aa), wild-type GroEL (1-544 aa), and GroEL mutants (GroEL-T21A, GroEL-T132A, and GroEL-T329A) were cloned into the E. coli, B. anthracis shuttle vector, pYS5 (modified to express Spectinomycin resistance gene ${ }^{63}$ ), and electroporated in B. anthracis Sterne strain (Bas-wt or Bas $\Delta$ prkC strain) (BTX Electro Cell Manipulator 600).

\section{GroEL complementation in E. coli SV2 strain}

Complementation study on $B$. anthracis GroEL variants was performed as described previously. ${ }^{36}$ Briefly, $B$. anthracis groEL variants and groES were cloned into pProEx-HTc and cloned in pACYCDuet-1, respectively. Individual groEL variants were co-expressed with groES in E. coli SV2. The cultures of $E$. coli SV2 expressing the required genes were serially diluted and spotted onto LB agar plates. The plates were incubated at permissive $\left(30^{\circ} \mathrm{C}\right)$ and restrictive $\left(42^{\circ} \mathrm{C}\right)$ conditions. Plasmid encoding E. coli GroES and GroEL, pSCM1603, was included as control. ${ }^{36}$

Expression and purification of recombinant proteins from $E$. coli The recombinant plasmids were transformed and proteins were overexpressed in E. coli BL-21 (DE3). The recombinant GST-tagged and $\mathrm{His}_{6^{-}}$ tagged fusion proteins were affinity purified with glutathione sepharose column (Qiagen, India) and $\mathrm{Ni}^{2+}$-NTA affinity column (Qiagen), respectively, as described previously. ${ }^{64}$

\section{In vitro kinase and phosphatase assays}

In vitro kinase assays (PrkC kinase/catalytic domain $\operatorname{PrkC}_{c}, 1 \mu \mathrm{g}$ ) were carried out in kinase buffer (20 mM HEPES pH 7.2, $10 \mathrm{mM} \mathrm{MgCl}_{2}$, and $10 \mathrm{mM} \mathrm{MnCl}_{2}$ ) containing $2 \mu \mathrm{Ci}\left[\gamma^{-32} \mathrm{P}\right] A T P$ (BRIT, Hyderabad, India) followed by incubation at $25^{\circ} \mathrm{C}$ for $30 \mathrm{~min}$ or as indicated in the text. Phosphorylation assays of substrates were carried out similarly, using $5 \mu \mathrm{g}$ substrates (Ef-Tu, Ef-G, SodA2, and GroEL). Reactions were terminated by $5 \times$ SDS sample buffer followed by boiling at $100^{\circ} \mathrm{C}$ for $5 \mathrm{~min}$. Proteins were separated by SDS-PAGE and analyzed by Personal Molecular Imager (PMI, BioRad). The images were quantitated by QuantityOne software (PMI, BioRad). Dephosphorylation was carried out by incubating the kinase reaction samples with Ser/Thr phosphatase PrpC $(1 \mu \mathrm{g})$ for additional $30 \mathrm{~min}$ at $37^{\circ} \mathrm{C}$, as described. ${ }^{14,} 22$

For time-dependent kinase assay, $\operatorname{PrkC}_{c}$ was first autophosphorylated (preactivated) using cold ATP. The autophosphorylated active kinase was then incubated with GroEL or SodA2 in kinase buffer containing $2 \mu \mathrm{Ci}$ $\left[\gamma^{-32}\right.$ P]ATP with increasing time points up to $30 \mathrm{~min}$. The phosphotransfer on substrates was observed and quantitated. 
Immunoblotting to identify phosphorylated residues

To detect the phosphorylated proteins, immunoblotting with $a$-pThr was performed as described previously. ${ }^{13}$ Proteins were resolved by SDS-PAGE and transferred onto a nitrocellulose membrane. Blots were exposed to $a-$ pThr antibody and goat anti-rabbit IgG secondary antibodies. The blots were developed by SuperSignal ${ }^{\circledR}$ West Pico Chemiluminescent Substrate kit (Pierce Protein Research Products), according to the manufacturer's instructions.

\section{Co-expression of GroEL with PrkC and PrpC}

Bas0253 cloned in pGEX-5X-3 or pProEx-HTc was co-expressed in E. coli BL21 (DE3) cells with pACYC-PrkC or pACYC-PrpC, to generate phosphorylated and unphosphorylated proteins, respectively. The co-expressed transformants were selected on ampicillin and chloramphenicol. The selected colonies were grown and maintained in media containing ampicillin and chloramphenicol and proteins were overexpressed with Isopropyl $\beta$-D-1-thiogalactopyranoside (IPTG). Phosphorylation status of these proteins was analyzed by Pro- $Q^{\circ}$ Diamond phospho-specific stain (Molecular Probes, Life Technologies) followed by SYPRO Ruby Protein Gel stain (Molecular Probes, Life Technologies) and Coomassie Brilliant Blue stain, according to the manufacturer's instructions. Pro-Q Diamond is a sensitive non-covalent fluorescent dye staining technology used for the detection of phosphoserine, phosphothreonine, and phosphotyrosine containing proteins. These proteins were used for subsequent assays.

\section{Recombinant GroEL size exclusion chromatography}

His $_{6}$-tagged GroEL-UP or GroEL-P were used for purification by size exclusion chromatography. Similar procedure was followed as described earlier. ${ }^{36}$ Briefly, the $\mathrm{Ni}^{2+}$-NTA purified proteins were dialyzed with $50 \mathrm{mM}$ Tris (pH 8.0), $150 \mathrm{mM} \mathrm{NaCl}$, and $1 \mathrm{mM}$ EDTA. The preparations were resolved on Superose $610 / 100$ GL (GE Healthcare) connected to the NGC Quest Plus Chromatography System (BioRad), with column volume of 23.6 $\mathrm{ml}$ and flow rate of $0.5 \mathrm{ml} / \mathrm{min}$. The protein standards on Superose 6 column were: Thyroglobulin $(660 \mathrm{kD})$, Ferritin $(440 \mathrm{kD}), \mathrm{BSA}(66 \mathrm{kD})$, and RNase $A(13.7 \mathrm{kD})$ that elute at $12,14,16.5$, and $18 \mathrm{ml}$, with partition coefficients $\left(K_{\mathrm{av}}\right)$ of $0.28,0.40,0.56$, and 0.72 , respectively.

\section{Proteinase $\mathrm{K}$ protection assay}

Protection of GroEL by GroES was performed as described earlier with minor modifications. ${ }^{65} \mathrm{His}_{6}$-tagged GroEL-UP and GroEL-P $(0.8 \mu \mathrm{M}$ each) were incubated for $10 \mathrm{~min}$ at $25^{\circ} \mathrm{C}$ in buffer A (10 mM MOPS-KOH [pH 7.2] and $50 \mathrm{mM} \mathrm{KCl}$ ) containing $5 \mathrm{mM} \mathrm{Mg}$-acetate, $1 \mathrm{mM} \mathrm{ADP}$, and $12.5 \mu \mathrm{M}$ $\mathrm{His}_{6}$-tagged GroES. The reactions were then cooled to $0{ }^{\circ} \mathrm{C}$ and incubated with proteinase $\mathrm{K}(12.5 \mu \mathrm{g} / \mathrm{ml})$. At times 0 and $2 \mathrm{~min}$, aliquots were removed and PMSF was added to a final concentration of $1 \mathrm{mM}$. The samples were then subjected to TCA precipitation and precipitates were resolved by SDS-PAGE and analyzed by immunoblotting using anti-GroEL antibodies.

\section{GroEL structure generation}

B. anthracis GroEL sequence was modeled using the co-ordinates from E. coli GroEL structure (PDB code: 1 AON chain A) with Modeller v9.13. The figures were generated in Pymol 1.3 as described earlier. ${ }^{66}$ Phosphorylated threonine residues are indicated as spheres in blue. Apical, intermediate, and equatorial domains are color coded in gold, silver, and green, respectively.

Few methods have been discussed in detail in Supplementary File.

\section{ACKNOWLEDGEMENTS}

We thank Jonathan Dworkin (Department of Microbiology and Immunology, College of Physicians and Surgeons, Columbia University, New York) for providing the $B$. subtilis (wild type, prkC deletion, and prkC complement) strains and prkC deletion strain of B. anthracis. We also thank Lakshmi Rajagopal (Department of Pediatric Infectious Diseases and Department of Obstetrics and Gynecology, University of Washington, School of Medicine, Seattle, Washington) for providing S. agalactiae strains and Prof. Rakesh Bhatnagar (Jawahar Lal Nehru University, New Delhi, India) for providing the $B$. anthracis GroEL-specific antibodies. We thank Katrin Bäsell (Institute of Microbiology, Ernst-Moritz-Arndt-University, Greifswald, Germany) for helping in the mass spectrometry-based phosphorylation site analysis. We thank Dr. Nirpendra Singh, UDSC, for his help in the mass spectrometry. This work was supported by Council of Scientific and Industrial Research (CSIR)-funded projects BSC-0123 and BSC-0104 and JC Bose Fellowship Research Grant (SERB) to Yogendra Singh.

\section{AUTHOR CONTRIBUTIONS}

G.A. and A. Sajid conceived and designed the experiments. A. Sajid, G.A., A. Singhal, R. V., C.M.S.K., N.D., T.K., A.M., R.M., V.M., and D.B. performed the experiments. A. Sajid and G.A. wrote the paper and prepared the figures. V.M., U.G., S.M., and Y.S. contributed reagents/materials/analysis tools. All authors have read and approved the manuscript.

\section{COMPETING INTERESTS}

The authors declare that they have no competing interest.

\section{REFERENCES}

1. Hanna, P. C. \& Ireland, J. A. Understanding Bacillus anthracis pathogenesis. Trends Microbiol. 7, 180-182 (1999).

2. Kolsto, A. B., Tourasse, N. J. \& Okstad, O. A. What sets Bacillus anthracis apart from other Bacillus species? Annu. Rev. Microbiol. 63, 451-476 (2009).

3. Lee, K. et al. Phenotypic and functional characterization of Bacillus anthracis biofilms. Microbiology 153, 1693-1701 (2007).

4. Li, Z., Hwang, S. \& Bar-Peled, M. Discovery of a unique extracellular polysaccharide in members of the pathogenic Bacillus that can co-form with spores. $J$. Biol. Chem. 36, 19051-19067 (2016).

5. Anand, A. et al. Polyketide quinones are alternate intermediate electron carriers during mycobacterial respiration in oxygen-deficient niches. Mol. Cell 4, 637-650 (2015).

6. Fagerlund, A. et al. Cyclic diguanylate regulation of Bacillus cereus group biofilm formation. Mol. Microbiol. 3, 471-494 (2016).

7. Koul, S., Prakash, J., Mishra, A. \& Kalia, V. C. Potential emergence of multi-quorum sensing inhibitor resistant (MQSIR) bacteria. Ind. J. Microbio. 1, 1-18 (2016).

8. Nguyen, D. et al. Active starvation responses mediate antibiotic tolerance in biofilms and nutrient-limited bacteria. Science 334, 982-986 (2011).

9. Auger, S. et al. Biofilm formation and cell surface properties among pathogenic and nonpathogenic strains of the Bacillus cereus group. Appl. Environ. Microbiol. 75, 6616-6618 (2009).

10. Branda, S. S. et al. Genes involved in formation of structured multicellular communities by Bacillus subtilis. J. Bacteriol. 186, 3970-3979 (2004).

11. Macfarlane, S. \& Dillon, J. F. Microbial biofilms in the human gastrointestinal tract. J. Appl. Microbiol. 102, 1187-1196 (2007).

12. Elsholz, A. K., Wacker, S. A. \& Losick, R. Self-regulation of exopolysaccharide production in Bacillus subtilis by a tyrosine kinase. Genes Dev. 15, 1710-1720 (2014).

13. Arora, G. et al. Unveiling the novel dual specificity protein kinases in Bacillus anthracis: identification of the first prokaryotic dual specificity tyrosine phosphorylationregulated kinase (DYRK)-like kinase. J. Biol. Chem. 287, 26749-26763 (2012).

14. Arora, G. et al. Zinc regulates the activity of kinase-phosphatase pair (BasPrkC) BasPrpC) in Bacillus anthracis. Biometals 26, 715-730 (2013).

15. Bryant-Hudson, K. M., Shakir, S. M. \& Ballard, J. D. Autoregulatory characteristics of a Bacillus anthracis serine/threonine kinase. J. Bacteriol. 193, 1833-1842 (2011).

16. Shakir, S. M. et al. Regulatory interactions of a virulence-associated serine/threonine phosphatase-kinase pair in Bacillus anthracis. J. Bacteriol. 192 400-409 (2010).

17. Shah, I. M., Laaberki, M. H., Popham, D. L. \& Dworkin, J. A. Eukaryotic-like Ser/Thr kinase signals bacteria to exit dormancy in response to peptidoglycan fragments. Cell 135, 486-496 (2008).

18. Shah, I. M. \& Dworkin, J. Induction and regulation of a secreted peptidoglycan hydrolase by a membrane Ser/Thr kinase that detects muropeptides. Mol. Microbiol. 75, 1232-1243 (2010).

19. Dworkin, J. Ser/Thr phosphorylation as a regulatory mechanism in bacteria. Curr. Opin. Microbiol. 24C, 47-52 (2015).

20. Pereira, S. F., Gonzalez, R. L. Jr. \& Dworkin, J. Protein synthesis during cellular quiescence is inhibited by phosphorylation of a translational elongation factor. Proc. Natl. Acad. Sci. U. S. A 112, E3274-E3281 (2015).

21. Sajid, A. et al. Interaction of Mycobacterium tuberculosis elongation factor Tu with GTP is regulated by phosphorylation. J. Bacteriol. 193, 5347-5358 (2011).

22. Sajid, A. et al. Phosphorylation of Mycobacterium tuberculosis Ser/Thr phosphatase by PknA and PknB. PLoS ONE 6, e17871 (2011).

23. Sajid, A., Arora, G., Singhal, A., Kalia, V. C. \& Singh, Y. Protein phosphatases of pathogenic bacteria: role in physiology and virulence. Annu. Rev. Microbiol. 69, 527-547 (2015). 
24. Donat, S. et al. Transcriptome and functional analysis of the eukaryotic-type serine/threonine kinase $\mathrm{PknB}$ in Staphylococcus aureus. J. Bacteriol. 191, 4056-4069 (2009).

25. Fleurie, A. et al. MapZ marks the division sites and positions FtsZ rings in Streptococcus pneumoniae. Nature 516, 259-262 (2014).

26. Schmidl, S. R. et al. The stability of cytadherence proteins in Mycoplasma pneumoniae requires activity of the protein kinase PrkC. Infect. Immun. 78, 184-192 (2010).

27. Chawla, Y. et al. Protein kinase B (PknB) of Mycobacterium tuberculosis is essential for growth of the pathogen in vitro as well as for survival within the host. J. Biol. Chem. 289, 13858-13875 (2014).

28. Sharma, A. et al. Serine/Threonine protein phosphatase PSTP of Mycobacterium tuberculosis is necessary for accurate cell division and survival of pathogen. J. Biol. Chem. 46, 24215-24230 (2016).

29. Absalon, C. et al. CpgA, EF-Tu and the stressosome protein YezB are substrates of the Ser/Thr kinase/phosphatase couple, PrkC/PrpC, in Bacillus subtilis. Microbiology 155, 932-943 (2009).

30. Lemos, J. A., Luzardo, Y. \& Burne, R. A. Physiologic effects of forced downregulation of dnaK and groEL expression in Streptococcus mutans. J. Bacteriol. 189, 1582-1588 (2007).

31. Ojha, A. et al. GroEL1: a dedicated chaperone involved in mycolic acid biosynthesis during biofilm formation in mycobacteria. Cell 123, 861-873 (2005).

32. Horwich, A. L., Farr, G. W. \& Fenton, W. A. GroEL-GroES-mediated protein folding. Chem. Rev. 106, 1917-1930 (2006).

33. Radford, S. E. GroEL: more than just a folding cage. Cell 125, 831-833 (2006).

34. Chakraborty, K. et al. Chaperonin-catalyzed rescue of kinetically trapped states in protein folding. Cell 1, 112-122 (2010).

35. Martin, J. GroEL/GroES interaction assayed by protease protection. Methods Mol. Biol. 140, 71-74 (2000).

36. Kumar, C. M. et al. Facilitated oligomerization of mycobacterial GroEL: evidence for phosphorylation-mediated oligomerization. J. Bacteriol. 191, 6525-6538 (2009).

37. Banu, L. D. et al. The Streptococcus mutans serine/threonine kinase, PknB, regulates competence development, bacteriocin production, and cell wall metabolism. Infect. Immun. 78, 2209-2220 (2010).

38. Gupta, M., Sajid, A., Arora, G., Tandon, V. \& Singh, Y. Forkhead-associated domaincontaining protein Rv0019c and polyketide-associated protein PapA5, from substrates of serine/threonine protein kinase $\mathrm{PknB}$ to interacting proteins of Mycobacterium tuberculosis. J. Biol. Chem. 284, 34723-34734 (2009).

39. Liu, Q. et al. The eukaryotic-type serine/threonine protein kinase STK is required for biofilm formation and virulence in Staphylococcus epidermidis. PLOS ONE 6, e25380 (2011).

40. Tamber, S., Schwartzman, J. \& Cheung, A. L. Role of PknB kinase in antibiotic resistance and virulence in community-acquired methicillin-resistant Staphylococcus aureus strain USA300. Infect. Immun. 78, 3637-3646 (2010).

41. Madec, E., Laszkiewicz, A., Iwanicki, A., Obuchowski, M. \& Seror, S. Characterization of a membrane-linked Ser/Thr protein kinase in Bacillus subtilis, implicated in developmental processes. Mol. Microbiol. 46, 571-586 (2002).

42. Chen, Y. et al. A Bacillus subtilis sensor kinase involved in triggering biofilm formation on the roots of tomato plants. Mol. Microbiol. 85, 418-430 (2012).

43. Vlamakis, H., Chai, Y., Beauregard, P., Losick, R. \& Kolter, R. Sticking together: building a biofilm the Bacillus subtilis way. Nat. Rev. Microbiol. 11, 157-168 (2013)

44. Leiba, J. et al. A novel mode of regulation of the Staphylococcus aureus catabolite control protein A (CcpA) mediated by Stk1 protein phosphorylation. J. Biol. Chem. 287, 43607-43619 (2012).

45. Reck, M. et al. The biofilm inhibitor carolacton disturbs membrane integrity and cell division of Streptococcus mutans through the serine/threonine protein kinase PknB. J. Bacteriol. 193, 5692-5706 (2011).

46. Kim, Y. E., Hipp, M. S., Bracher, A., Hayer-Hartl, M. \& Hartl, F. U. Molecular chaperone functions in protein folding and proteostasis. Annu. Rev. Biochem. 82, 323-355 (2013).

47. Sinha, K. \& Bhatnagar, R. GroEL provides protection against Bacillus anthracis infection in BALB/c mice. Mol. Immunol. 48, 264-271 (2010).
48. Sinha, K. \& Bhatnagar, R. Recombinant GroEL enhances protective antigenmediated protection against Bacillus anthracis spore challenge. Med. Microbiol. Immunol. 202, 153-165 (2013).

49. Sherman, M. \& Goldberg, A. L. Heat shock-induced phosphorylation of GroEL alters its binding and dissociation from unfolded proteins. J. Biol. Chem. 269, 31479-31483 (1994)

50. Sherman, M. Y. \& Goldberg, A. L. Heat shock in Escherichia coli alters the proteinbinding properties of the chaperonin groEL by inducing its phosphorylation. Nature 357, 167-169 (1992).

51. Lee, N. K., Yeo, I. C., Park, J. W. \& Hahm, Y. T. Growth inhibition and induction of stress protein, GroEL, of Bacillus cereus exposed to antibacterial peptide isolated from Bacillus subtilis SC-8. Appl. Biochem. Biotechnol. 165, 235-242 (2011).

52. Susin, M. F., Baldini, R. L., Gueiros-Filho, F. \& Gomes, S. L. GroES/GroEL and DnaK/ DnaJ have distinct roles in stress responses and during cell cycle progression in Caulobacter crescentus. J. Bacteriol. 188, 8044-8053 (2006).

53. Chen, J. et al. Fibrillogenic propensity of the GroEL apical domain: a Janus-faced minichaperone. FEBS Lett. 586, 1120-1127 (2012).

54. Gallaher, T. K., Wu, S., Webster, P. \& Aguilera, R. Identification of biofilm proteins in non-typeable Haemophilus influenzae. BMC Microbiol. 6, 65 (2006).

55. Kalmokoff, M. et al. Proteomic analysis of Campylobacter jejuni 11168 biofilms reveals a role for the motility complex in biofilm formation. J. Bacteriol. 188, 4312-4320 (2006)

56. Klein, M. I. et al. Streptococcus mutans protein synthesis during mixed-species biofilm development by high-throughput quantitative proteomics. PLOS ONE 7, e45795 (2012).

57. Bergonzelli, G. E. et al. GroEL of Lactobacillus johnsonii La1 (NCC 533) is cell surface associated: potential role in interactions with the host and the gastric pathogen Helicobacter pylori. Infect. Immun. 74, 425-434 (2006).

58. Chung, M. C. et al. Bacillus anthracis interacts with plasmin(ogen) to evade C3bdependent innate immunity. PLOS ONE 6, e18119 (2011).

59. Joshi, S., Bisht, G. S., Rawat, D. S., Maiti, S. \& Pasha, S. Comparative mode of action of novel hybrid peptide CS-1a and its rearranged amphipathic analogue CS-2a. FEBS J. 279, 3776-3790 (2012).

60. Singhal, A. et al. Systematic analysis of mycobacterial acylation reveals first example of acylation-mediated regulation of enzyme activity of a bacterial phosphatase. J. Biol. Chem. 290, 26218-26234 (2015).

61. Auger, S., Krin, E., Aymerich, S. \& Gohar, M. Autoinducer 2 affects biofilm formation by Bacillus cereus. Appl. Environ. Microbiol. 72, 937-941 (2006).

62. Schmidl, S. R. et al. The phosphoproteome of the minimal bacterium Mycoplasma pneumoniae: analysis of the complete known Ser/Thr kinome suggests the existence of novel kinases. Mol. Cell. Proteomics 9, 1228-1242 (2010).

63. Singh, L. K. et al. clpC operon regulates cell architecture and sporulation in Bacillus anthracis. Environ. Microbiol. 17, 855-865 (2015).

64. Gupta, M. et al HupB, a nucleoid-associated protein of Mycobacterium tuberculosis, is modified by Serine/Threonine protein kinases in vivo. J. Bacteriol. 196, 2646-2657 (2014).

65. Langer, T., Pfeifer, G., Martin, J., Baumeister, W. \& Hartl, F. U. Chaperonin-mediated protein folding: GroES binds to one end of the GroEL cylinder, which accommodates the protein substrate within its central cavity. EMBO J. 11, 4757-4765 (1992).

66. Arora, G. et al. Identification of Ser/Thr kinase and forkhead associated domains in Mycobacterium ulcerans: characterization of novel association between protein kinase Q and MupFHA. PLoS Negl. Trop. Dis. 8, e3315 (2014).

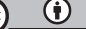

This work is licensed under a Creative Commons Attribution 4.0 International License. The images or other third party material in this article are included in the article's Creative Commons license, unless indicated otherwise in the credit line; if the material is not included under the Creative Commons license, users will need to obtain permission from the license holder to reproduce the material. To view a copy of this license, visit http://creativecommons.org/licenses/by/ 4.0

(c) The Author(s) 2017

Supplementary Information accompanies the paper on the npj Biofilms and Microbiomes website (doi:10.1038/s41522-017-0015-4). 